\title{
The Incidence and Risk Analysis of Lung Cancer Development in Patients with Chronic Obstructive Pulmonary Disease: Possible Effectiveness of Annual CT-Screening
}

This article was published in the following Dove Press journal:

International Journal of Chronic Obstructive Pulmonary Disease

Hiroyoshi Machida'
Sumito Inoue'
Yoko Shibata $\mathbb{D}^{2}$
Tomomi Kimura
Takahito Ota'
Yu Ishibashi'
Hiroaki Murano'
Kodai Furuyama'
Sujeong Yang'
Hiroshi Nakano'
Kento Sato'
Masamichi Sato'
Takako Nemoto'
Michiko Nishiwaki'
Keiko Yamauchi'
Akira Igarashi'
Masafumi Watanabe'
'Department of Cardiology,
Pulmonology, and Nephrology, Yamagata
University Faculty of Medine, Yamagata,
Japan; 'Department of Pulmonary
Medicine, Fukushima Medical University,
Fukushima, Japan; ${ }^{3}$ Respiratory Medicine,
Yamagata Saisei Hospital, Yamagata, Japan

Correspondence: Sumito Inoue Department of Cardiology, Pulmonology, and Nephrology, Yamagata University Faculty of Medicine, 2-2-2 lida-Nishi, Yamagata, 990-9585, Japan

Email sinoue@med.id.yamagata-u.ac.jp

\begin{abstract}
Purpose: Lung cancer is a serious complication in patients with chronic obstructive pulmonary disease (COPD) and accounts for approximately $15 \%$ of deaths in patients with COPD. However, with the exception of emphysema, few reports to date have been published on the factors that predict lung cancer development in COPD patients. It has been reported that patients with COPD develop lung cancer at a rate of $0.8 \%-1.7 \%$ year, but the incidence may be higher in the Japanese population. Therefore, we investigated the incidence of lung cancer and the lung cancer mortality rate in Japanese COPD patients, as well as factors that are associated with the development of lung cancer in COPD patients.
\end{abstract}

Patients and Methods: We followed up 224 patients with stable COPD and performed CT examinations at least once per year. The incidence of lung cancer was recorded and data at enrollment were compared with data of the group that did not develop lung cancer.

Results: Over a median follow-up period of 4.58 years, lung cancer was newly diagnosed in 19 patients; the incidence of lung cancer in this population was $1.85 \%$ /year. Patients who developed lung cancer had more severe emphysema assessed by CT and GOLD classification and were more likely to be current smokers than those who did not develop lung cancer. No other significant differences were observed between these two groups. Mortality was significantly increased in patients who developed lung cancer compared with those who did not.

Conclusion: In COPD patients, the incidence of lung cancer is higher and the development of lung cancer worsens the prognosis; however, lung cancer development is unpredictable and attention should be paid to all patients. Annual CT screening is important for early detection of lung cancer.

Keywords: chronic obstructive pulmonary disease, COPD, lung cancer, GOLD classification, current smoker, inhaled corticosteroid, ICS

\section{Plain Language Summary}

The main contribution of our study is the incidence of lung cancer in higher and the development of lung cancer worsens the prognosis of patients with chronic obstructive pulmonary disease (COPD). It was difficult to predict the development of lung cancer in patients with COPD, we need to pay attention to the development of lung cancer in all COPD patients. Annual CT screening is important for early detection of lung cancer. 


\section{Introduction}

Chronic obstructive pulmonary disease (COPD) is a major and increasing global health problem and is the third leading cause of death worldwide. ${ }^{1}$ COPD is an inflammatory disease of the lungs that is caused by long-term exposure to noxious particles and gases, especially cigarette smoke. ${ }^{2}$ Airflow obstruction, emphysema, chronic bronchitis, and lung cancer are types of lung damage associated with cigarette smoking. ${ }^{3}$ There is sufficient evidence that an important complication of COPD is in its correlation with lung cancer. ${ }^{4}$ In addition, COPD is a risk factor for lung cancer development independent of smoking status. COPD patients are three to six times more likely to develop lung cancer than smokers without COPD, ${ }^{5,6}$ and it has been reported that COPD patients develop lung cancer at a rate of $0.8-1.7 \% /$ year. ${ }^{7,8}$ On the contrary, a three-year observational study in Japan recently reported that the incidence of lung cancer in COPD patients was $2.30 \%$ /year. ${ }^{9}$ Similarly, according to an eightyear observational study conducted in Japan, Haruna et al reported that the incidence of lung cancer was $2.06 \%$ / year. ${ }^{10}$

Complications involving malignant neoplasms such as lung cancer greatly affect the quality of life and prognosis of COPD patients. ${ }^{11}$ Lung cancer reportedly accounts for $12 \%$ to $14 \%$ of COPD-related deaths. ${ }^{12-14}$ However, although the percentage varies between studies, some studies have found that lung cancer accounts for $33 \%$ of all COPD-related deaths. ${ }^{15}$ It has also been reported that lung cancer accounts for approximately $15 \%$ of COPD deaths in Japan. ${ }^{10,16}$

The degree of emphysematous lesions has been reported to be associated with lung cancer development in COPD patients. ${ }^{9,17,18}$ One study reported an increased risk of lung cancer as COPD airflow limitations progressed. ${ }^{19}$ In contrast, a recent study stated that patients with milder stages of COPD are at higher risk for lung cancer than those with moderate or severe COPD. ${ }^{20}$ Based on these studies, the finding that the risk of lung cancer is dependent on the severity of airflow limitation is controversial. In addition, asthma complications ${ }^{21}$ and inhaled corticosteroid (ICS) use $\mathrm{e}^{21,22}$ have recently been shown to be associated with lower lung cancer rates in patients with COPD. However, no evidence has been found to support the idea that asthma complications or the use of ICS actually suppress lung cancer development in COPD patients.
As mentioned above, lung cancer is often associated with COPD and has a significant prognostic impact. However, with the exception of emphysema, few studies to date have reported the factors that predict lung cancer development in COPD patients. In addition, the lung cancer incidence in COPD patients in Japan may be higher than previously thought. Therefore, we investigated lung cancer incidence and mortality in COPD patients as well as factors associated with lung cancer development in this patient population.

\section{Patients and Methods Study Population}

The Institutional Ethics Committee of the Yamagata University Faculty of Medicine approved the research protocol (approval number; H23-134, approval date January 5, 2012). Written informed consent was obtained from all patients prior to participation. This study was performed according to the principles outlined in the Declaration of Helsinki. We enrolled 253 stable COPD patients who presented to the Yamagata University Hospital outpatient department from January 2014 to January 2020 and performed follow-up until April 2020. All participants were 40 years of age or older and had a previous or current smoking history of at least 10 pack-years. Each participant's COPD diagnosis was based on the Global Initiative for Chronic Obstructive Lung Disease guideline standards (forced expiratory volume during the first second of forced breath $\left[\mathrm{FEV}_{1}\right]$ to forced vital capacity $[\mathrm{FVC}]$ ratio after bronchodilation $<0.70$ ). Participants with asthma complications or participants who were previously diagnosed with asthma were also included in this study. Each participant's asthma and COPD overlap (ACO) diagnosis were based on The Japanese Respiratory Society (JRS) Guidelines for the Management of ACO 2018. ${ }^{23}$ According to this guideline, we diagnosed as ACO when the patients with COPD had at least one of [1][3] and at least two of [4]. [1]: Variable or paroxysmal clinical symptoms, [2]: Previous diagnosis of asthma before 40 years-old, [3]: Increase of fractional exhaled nitric oxide (FeNO) $>35 \mathrm{ppb}$, [4]: 1) A history of allergic rhinitis, 2) Airway reversibility (improvement of $\mathrm{FEV}_{1}$ is $>12 \%$ and $>200 \mathrm{~mL}$ after inhalation of bronchodilator), 3 ) Elevated peripheral eosinophils $(>5 \%$ or $>300 / \mu \mathrm{L}), 4)$ Elevated total or allergen-specific immunoglobulin (Ig) E level. 


\section{Study Protocol}

At the time of registration, sex, age, BMI, smoking status, treatment history, and symptoms, including the CAT score, were recorded. In addition, at the time of registration, spirometry was performed both before and after inhalation of a bronchodilator, and a blood test and chest CT were also performed. The treatment course was determined by the attending physician and was not limited to specific treatments. While most participants visited the outpatient clinic once a month or once every two months, the frequency of outpatient visits was also determined by the attending physician; more stable participants visited the clinic once every three months or once every six months. At each outpatient visit, smoking status, medication status, and disease exacerbations were confirmed. Unplanned visits and hospital admissions were also recorded.

\section{Pulmonary Function Tests}

Spirometry before and after inhalation of a bronchodilator (salbutamol) was performed once every six months. For participants who were subjected to spirometry for more than two years (at least three times), individual yearly changes in $\mathrm{FEV}_{1}$ after inhalation of bronchodilators and the percentage of predicted $\mathrm{FEV}_{1}\left(\% \mathrm{FEV}_{1}\right)$ after inhalation of bronchodilators were calculated.

\section{Image Evaluation}

CT examinations were performed at least once per year. CT was also performed if the chest $\mathrm{x}$-ray findings were abnormal or if a CT scan was required to verify the presence of co-morbidities. Although the intervals for chest radiographs were not specified, most participants received a chest $\mathrm{x}$-ray at least once every six months. CT evaluation for emphysema was performed using LungVision $^{\circledR}$ (CYBERNET). On chest CT, we evaluated the low attenuation area (LAA) by visualizing in yellow color, and scored $(0-4 ; 0$ : less than $5 \%$ of lung field, $1: 5 \%$ or more and less than $25 \%$ of the lung field, $2: 25 \%$ or more and less than $50 \%$ of the lung field, $3: 50 \%$ or more and less than $75 \%$ of the lung field, 4 : over $75 \%$ of the lung field). Upper, middle and lower parts of both lung fields were measured and scored $(0-24 ; 0-7$ as mild, $8-15$ as Moderate, 16 - as severe). ${ }^{24}$

\section{Statistical Analyses}

Data are presented as the mean \pm standard deviation (SD), the median (range in parentheses, 25th to 75th percentile), or as n. A univariate analysis was performed using the Chi-square test, Student's $t$-test, or the Wilcoxon rank sum test where appropriate. All statistical analyses were performed using $\mathrm{JMP}^{\circledR}$ (ver13).

\section{Results}

Figure 1 illustrates the outline of this study. Of the enrolled patients, eight were excluded from the analysis due to missing blood data, while two were excluded due to missing Asthma-COPD overlap (ACO) data. Eight patients were excluded because of a short follow-up period (less than six months). Additionally, nine patients were excluded because they had a history of lung cancer, and two patients were excluded because they had lung cancer at enrollment. Therefore, we analyzed 224 patients with stable COPD.

Table 1 lists the characteristics of the patients included in this study. The average age was $70.4 \pm 8.4$ years, and most patients were male. The median follow-up period was 4.58 (range, 2.91-6.25) years, during which 19 patients developed lung cancer. Therefore, the incidence of lung cancer was $1.85 \%$ year.

Table 2 shows a comparison of the characteristics of patients who developed lung cancer with those of patients who did not. Age, body mass index (BMI), COPD

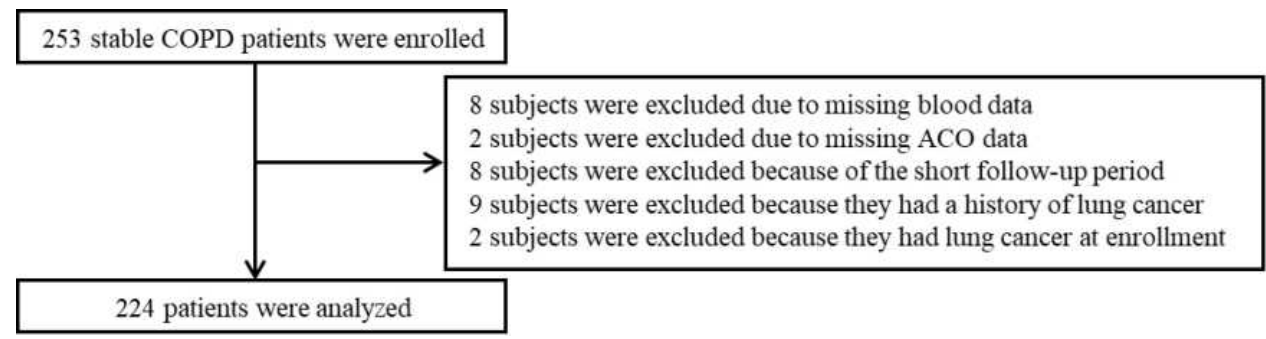

Figure I Study outline. We enrolled 253 stable COPD patients. Of the enrolled patients, eight were excluded from the analysis due to missing blood data, while two were excluded due to missing Asthma-COPD overlap (ACO) data. Eight patients were excluded because of a short follow-up period (less than six months). Additionally, nine patients were excluded because they had a history of lung cancer, and two patients were excluded because they had lung cancer at enrollment. Therefore, we analyzed 224 patients with stable COPD. 
Table I Patient Characteristics

\begin{tabular}{|c|c|c|c|}
\hline & $\begin{array}{l}\text { Mean, } \\
\text { Number, or } \\
\text { Median } \\
\text { (Interquartile } \\
\text { Range) }\end{array}$ & & $\begin{array}{l}\text { Mean, } \\
\text { Number, or } \\
\text { Median } \\
\text { (Interquartile } \\
\text { Range) }\end{array}$ \\
\hline Age $(y r)$ & $70.4 \pm 8.4$ & $\mathrm{TP}(\mathrm{g} / \mathrm{dl})$ & $7.1 \pm 0.5$ \\
\hline Male/Female & $214 / 10$ & $\begin{array}{l}\text { T. Bil } \\
(\mathrm{mg} / \mathrm{dl})\end{array}$ & $0.74 \pm 0.31$ \\
\hline BMI $\left(\mathrm{kg} / \mathrm{m}^{2}\right)$ & $22.7 \pm 3.4$ & $\begin{array}{l}\text { AST (U/ } \\
\text { I) }\end{array}$ & $23.0 \pm 8.3$ \\
\hline $\begin{array}{l}\text { Follow-up } \\
\text { period (yr) }\end{array}$ & $4.58(2.91-6.25)$ & $\mathrm{ALT}(\mathrm{U} / \mathrm{l})$ & $21.6 \pm 11.2$ \\
\hline $\begin{array}{l}\text { Lung cancer } \\
\text { onset (\%/yr) }\end{array}$ & 1.85 & $\begin{array}{l}\mathrm{LDH}(\mathrm{U} / \\
\mathrm{I})\end{array}$ & $197.4 \pm 40.3$ \\
\hline Pack-years & $50.7 \pm 31.6$ & $\begin{array}{l}\text { BUN } \\
(\mathrm{mg} / \mathrm{dl})\end{array}$ & $16.9 \pm 5.2$ \\
\hline $\begin{array}{l}\text { Current } \\
\text { smoker (\%) }\end{array}$ & 12.9 & $\begin{array}{l}\mathrm{Cr}(\mathrm{mg} / \\
\mathrm{dl})\end{array}$ & $0.83 \pm 0.24$ \\
\hline Death (\%) & 8.0 & $\begin{array}{l}\text { eGFR } \\
(\mathrm{mL} / \mathrm{min} / \\
\left.1.73 \mathrm{~m}^{2}\right)\end{array}$ & $74.7 \pm 19.7$ \\
\hline $\begin{array}{l}\text { Survival period } \\
\text { (days) }\end{array}$ & $\begin{array}{l}167 \mid(1062 \\
-2282)\end{array}$ & $\begin{array}{l}\text { UA (mg/ } \\
\text { dl) }\end{array}$ & $5.9 \pm 1.4$ \\
\hline CAT (score) & $9.6 \pm 7.3$ & $\begin{array}{l}\mathrm{Na} \\
(\mathrm{mEq} / \mathrm{l})\end{array}$ & $14 \mid .3 \pm 2.3$ \\
\hline LAA (score) & $5.2 \pm 4.1$ & $\begin{array}{l}\mathrm{K}(\mathrm{mEq} / \\
\mathrm{l})\end{array}$ & $4.3 \pm 0.4$ \\
\hline $\begin{array}{l}\text { Change in LAA } \\
\text { (score) }\end{array}$ & $0.0(0.0-0.5)$ & $\begin{array}{l}\mathrm{Cl}(\mathrm{mEq} / \\
\mathrm{l})\end{array}$ & $104.7 \pm 2.6$ \\
\hline $\begin{array}{l}\text { Exacerbations } \\
\text { (/yr) }\end{array}$ & $0.00(0.00-0.16)$ & $\begin{array}{l}\text { WBC } \\
(/ \mu \mathrm{L})\end{array}$ & $6384.4 \pm 1960.7$ \\
\hline GOLD I/II/III/IV & $73 / 108 / 33 / 10$ & $\begin{array}{l}\text { Neut } \\
(/ \mu \mathrm{L})\end{array}$ & $3830.1 \pm 1470.1$ \\
\hline $\begin{array}{l}\text { GOLD A/B/C/ } \\
\text { D }\end{array}$ & $124 / 87 / 5 / 8$ & $\begin{array}{l}\text { Lymph (/ } \\
\mu \mathrm{L})\end{array}$ & $1816.9 \pm 685.3$ \\
\hline FVC (L) & $3.25 \pm 0.77$ & $\begin{array}{l}\text { Mono } \\
(/ \mu L)\end{array}$ & $392.8 \pm 142.1$ \\
\hline $\mathrm{FEV}_{\text {। }}(\mathrm{L})$ & $1.88 \pm 0.64$ & Eos $(/ \mu \mathrm{L})$ & $\begin{array}{l}170.0 \\
(100.0-260.0)\end{array}$ \\
\hline$\% F E V_{1}(\%)$ & $68.4 \pm 20.4$ & $\begin{array}{l}\text { Baso } \\
(/ \mu \mathrm{L})\end{array}$ & $41.7 \pm 23.4$ \\
\hline
\end{tabular}

(Continued)
Table I (Continued).

\begin{tabular}{|c|c|c|c|}
\hline & $\begin{array}{l}\text { Mean, } \\
\text { Number, or } \\
\text { Median } \\
\text { (Interquartile } \\
\text { Range) }\end{array}$ & & $\begin{array}{l}\text { Mean, } \\
\text { Number, or } \\
\text { Median } \\
\text { (Interquartile } \\
\text { Range) }\end{array}$ \\
\hline $\begin{array}{l}\text { Changes in } \\
\mathrm{FEV}_{\mathrm{I}}(\mathrm{mL} / \mathrm{yr})\end{array}$ & $\begin{array}{l}-14.0 \\
(-52.0-15.0)\end{array}$ & $\begin{array}{l}\text { RBC } \\
\left(10^{6} / \mu \mathrm{L}\right)\end{array}$ & $4.57 \pm 0.53$ \\
\hline $\begin{array}{l}\text { Changes in } \\
\% F E V_{1}(\% / y r)\end{array}$ & $\begin{array}{l}0.112 \\
(-1.177-1.375)\end{array}$ & $\mathrm{Hb}(\mathrm{g} / \mathrm{dl})$ & $14.0 \pm 1.6$ \\
\hline $\begin{array}{l}\text { Bronchodilator } \\
\text { reversibility (\%) }\end{array}$ & 15.8 & $\mathrm{Ht}(\%)$ & $42.9 \pm 4.3$ \\
\hline FeNO (ppb) & $32.4 \pm 24.2$ & $\begin{array}{l}\text { Plt (104/ } \\
\mu L)\end{array}$ & $22.2 \pm 6.9$ \\
\hline $\begin{array}{l}\text { Use of LAMA } \\
\text { (\%) }\end{array}$ & 23.2 & $\begin{array}{l}\operatorname{lgE}(\mathrm{IU} / \\
\mathrm{mL})\end{array}$ & $\begin{array}{l}70.0 \\
(23.8-236.0)\end{array}$ \\
\hline $\begin{array}{l}\text { Use of LABA } \\
\text { (\%) }\end{array}$ & 20.1 & $\begin{array}{l}\text { Fe }(\mu g / \\
\text { dl) }\end{array}$ & $93.9 \pm 38.7$ \\
\hline $\begin{array}{l}\text { Use of ICS at } \\
\text { enrollment (\%) }\end{array}$ & 11.2 & $\begin{array}{l}\text { Fer (ng/ } \\
\mathrm{mL})\end{array}$ & $\begin{array}{l}94.0 \\
(44.0-169.8)\end{array}$ \\
\hline $\begin{array}{l}\text { Use of ICS } \\
\text { during follow- } \\
\text { up period (\%) }\end{array}$ & 29.9 & $\begin{array}{l}\text { hsCRP } \\
(\mathrm{mg} / \mathrm{dl})\end{array}$ & $0.09(0.04-0.21)$ \\
\hline $\mathrm{ACO}(\%)$ & 13.8 & $\begin{array}{l}\text { D-dimer } \\
(\mu g / d l)\end{array}$ & $0.83(0.54-1.39)$ \\
\hline $\begin{array}{l}\text { Interstitial } \\
\text { pneumonia (\%) }\end{array}$ & 7.6 & $\begin{array}{l}\text { Fib (mg/ } \\
\text { dl) }\end{array}$ & $329.0 \pm 77.5$ \\
\hline \multirow[t]{5}{*}{ CPFE (\%) } & 5.8 & $\begin{array}{l}5-\mathrm{HIAA} \\
(\mathrm{ng} / \mathrm{mL})\end{array}$ & $5.8(4.6-8.5)$ \\
\hline & & $\begin{array}{l}\text { ACE (U/ } \\
L)\end{array}$ & $12.8(10.0-15.5)$ \\
\hline & & $\begin{array}{l}\text { TARC } \\
(\mathrm{pg} / \mathrm{mL})\end{array}$ & $384.9 \pm 226.5$ \\
\hline & & $\begin{array}{l}\text { Hcy } \\
(\mathrm{nmol} / \\
\mathrm{mL})\end{array}$ & $11.4 \pm 5.1$ \\
\hline & & $\begin{array}{l}\text { PRA (ng/ } \\
\mathrm{mL} / \mathrm{hr})\end{array}$ & $1.2(0.7-2.1)$ \\
\hline
\end{tabular}

Note: Data are presented as mean $\pm S D$, median (with ranges in parentheses, 25th-75th percentile) or $n$.

Abbreviations: BMI, body mass index; CAT, COPD assessment score; LAA, low attenuation area on CT scan; FeNO, fractional exhaled nitric oxide; LAMA, long-acting muscarinic antagonist; LABA, long-acting $\beta 2$ agonist; ICS, inhaled corticosteroid; ACO, asthma-COPD overlap; CPFE, combined pulmonary fibrosis and emphysema; IgE, immunoglobulin E; Fer, ferritin; hsCRP, high-sensitivity C-reactive protein; Fib, fibrinogen; 5-HIAA, 5-hydroxyindoleacetic acid; TARC, thymus and activation-regulated chemokine; Hcy, homocysteine; PRA, plasma renin activity. 
assessment score (CAT), frequency of exacerbations, pulmonary function, changes in pulmonary function, treatment, and laboratory data were similar in both groups. Patients who developed lung cancer had more severe emphysema assessed by CT. The number of pack-years tended to be higher in the lung cancer development group, which was also composed of significantly higher numbers of current smokers at enrollment. In the lung cancer development group, the degree of airflow obstruction, as assessed by the GOLD classification, was more severe. The frequency of ACO tended to be higher in patients who did not develop lung cancer, and those patients also tended to have a higher rate of ICS use during the followup period. The incidence of interstitial pneumonia tended to be higher in the lung cancer development group, but no significant difference was observed. The proportion of basophils was also significantly lower in the lung cancer development group.

Next, we investigated the survival rate of all patients. Eighteen patients died during the follow-up period: seven patients in the lung cancer development group (36.8\%) and 11 patients in the group that did not develop lung cancer (5.4\%). The overall mortality rate during follow-up was significantly higher in the lung cancer group (Table $2, P<0.001$ ). A survival curve analysis according to the Kaplan-Meier method showed that mortality was significantly increased in the lung cancer development group compared with the group that did not develop lung cancer (Figure 2, Hazard ratio: $10.28 \%, 95 \%$ confidence interval $[\mathrm{CI}]: \quad 3.38-31.30$ $P<0.0001)$.

Table 3 lists the characteristics of the participants who developed lung cancer. The median time to develop lung cancer was 929 (range, 217-1042) days. Twelve patients were histologically diagnosed with lung cancer. Among the histological types, adenocarcinoma was the most common (50.0\%). Except for one patient, non-small cell lung cancer was detected at an early stage (Stage I, lung cancer was staged according to The Japan Lung Cancer Society criteria). ${ }^{25}$ NCSLC patients received the appropriate therapy, and none of those patients died during the follow-up period. Small cell lung cancer was detected at an advanced stage, and one patient died of the disease during follow-up, while another died of interstitial pneumonia. Two of the patients clinically diagnosed with lung cancer received radiation therapy, but both died during the follow-up period. The remaining five patients who were clinically diagnosed with lung cancer had elected to receive the best supportive care either
Table 2 Comparison of the Characteristics of Patients Who Developed Lung Cancer with Those Who Did Not

\begin{tabular}{|c|c|c|c|}
\hline & $\begin{array}{l}\text { Developed } \\
\text { Lung Cancer } \\
(n=19)\end{array}$ & $\begin{array}{l}\text { Did Not Develop } \\
\text { Lung Cancer } \\
(n=205)\end{array}$ & $P$ value \\
\hline Age (yr) & $72.4 \pm 7.1$ & $70.2 \pm 8.5$ & 0.2903 \\
\hline Male/Female & $19 / 0$ & $195 / 10$ & 0.3246 \\
\hline BMI $\left(\mathrm{kg} / \mathrm{m}^{2}\right)$ & $21.9 \pm 2.5$ & $22.7 \pm 3.5$ & 0.3509 \\
\hline $\begin{array}{l}\text { Follow-up } \\
\text { period }(\mathrm{yr})\end{array}$ & $\begin{array}{l}4.39 \\
(3.10-5.73)\end{array}$ & $4.6 I(2.7 I-6.26)$ & 0.7708 \\
\hline Pack-years & $62.7 \pm 31.0$ & $49.6 \pm 31.5$ & 0.0836 \\
\hline $\begin{array}{l}\text { Current smoker } \\
\text { (\%) }\end{array}$ & 31.6 & 11.2 & 0.0226 \\
\hline Death (\%) & 36.8 & 5.4 & $<0.001$ \\
\hline $\begin{array}{l}\text { Survival period } \\
\text { (days) }\end{array}$ & $\begin{array}{l}1602 \\
(|133-209|)\end{array}$ & $1682(99 \mid-2282)$ & 0.7708 \\
\hline CAT (score) & $10.4 \pm 6.3$ & $9.5 \pm 7.3$ & 0.6113 \\
\hline LAA (score) & $7.4 \pm 4.4$ & $5.1 \pm 4.1$ & 0.0437 \\
\hline $\begin{array}{l}\text { Change in LAA } \\
\text { (score) }\end{array}$ & $0.0(-0.3-0.5)$ & $0.0(0.0-0.5)$ & 0.7312 \\
\hline $\begin{array}{l}\text { Exacerbations } \\
(/ y r)\end{array}$ & $\begin{array}{l}0.00 \\
(0.00-0.25)\end{array}$ & $0.00(0.00-0.16)$ & 0.7128 \\
\hline GOLD I/II/III/IV & $7 / 7 / 1 / 4$ & $66 / 101 / 32 / 6$ & 0.0021 \\
\hline GOLD A/B/C/D & $7 / 11 / 0 / 1$ & $117 / 76 / 5 / 7$ & 0.2774 \\
\hline FVC (L) & $3.27 \pm 0.68$ & $3.24 \pm 0.78$ & 0.8808 \\
\hline $\mathrm{FEV}_{\text {। }}(\mathrm{L})$ & $1.78 \pm 0.81$ & $1.89 \pm 0.62$ & 0.4724 \\
\hline$\% \mathrm{FEV}_{\text {। }}(\%)$ & $63.8 \pm 26.9$ & $68.8 \pm 19.7$ & 0.3116 \\
\hline $\begin{array}{l}\text { Changes in FEV } \\
(\mathrm{mL} / \mathrm{yr})\end{array}$ & $\begin{array}{l}-21.0 \\
(-50.5-8.8)\end{array}$ & $-10.5(-52.3-25.8)$ & 0.4773 \\
\hline $\begin{array}{l}\text { Changes in } \\
\% F E V_{1}(\% / y r)\end{array}$ & $\begin{array}{l}-0.323 \\
(-1.132-1.407)\end{array}$ & $\begin{array}{l}0.220 \\
(-1.129-1.661)\end{array}$ & 0.6677 \\
\hline $\begin{array}{l}\text { Bronchodilator } \\
\text { reversibility (\%) }\end{array}$ & 7.1 & 16.4 & 0.7028 \\
\hline $\mathrm{FeNO}(\mathrm{ppb})$ & $25.5 \pm 11.7$ & $33.0 \pm 24.8$ & 0.2814 \\
\hline $\begin{array}{l}\text { Use of LAMA } \\
\text { (\%) }\end{array}$ & 5.3 & 24.9 & 0.0835 \\
\hline $\begin{array}{l}\text { Use of LABA } \\
\text { (\%) }\end{array}$ & 10.5 & 21.0 & 0.3780 \\
\hline $\begin{array}{l}\text { Use of ICS at } \\
\text { enrollment (\%) }\end{array}$ & 5.3 & 11.7 & 0.7031 \\
\hline
\end{tabular}

(Continued) 
Table 2 (Continued).

\begin{tabular}{|c|c|c|c|}
\hline & $\begin{array}{l}\text { Developed } \\
\text { Lung Cancer } \\
(n=19)\end{array}$ & $\begin{array}{l}\text { Did Not Develop } \\
\text { Lung Cancer } \\
(n=205)\end{array}$ & $P$ value \\
\hline $\begin{array}{l}\text { Use of ICS } \\
\text { during follow-up } \\
\text { period (\%) }\end{array}$ & 15.8 & 31.2 & 0.1599 \\
\hline $\mathrm{ACO}(\%)$ & 5.3 & 14.6 & 0.4843 \\
\hline $\begin{array}{l}\text { Interstitial } \\
\text { pneumonia (\%) }\end{array}$ & 15.8 & 6.8 & 0.1583 \\
\hline TP (g/dl) & $7.1 \pm 0.5$ & $7.1 \pm 0.5$ & 0.7975 \\
\hline T. Bil (mg/dl) & $0.74 \pm 0.41$ & $0.74 \pm 0.30$ & 0.9821 \\
\hline AST (U/l) & $22.2 \pm 12.5$ & $23.1 \pm 7.8$ & 0.6260 \\
\hline ALT (U/l) & $19.7 \pm 16.0$ & $21.8 \pm 10.7$ & 0.4446 \\
\hline LDH (U/I) & $182.0 \pm 27.7$ & $198.9 \pm 41.0$ & 0.0809 \\
\hline BUN (mg/dl) & $16.8 \pm 5.0$ & $16.9 \pm 5.2$ & 0.9465 \\
\hline Crea $(\mathrm{mg} / \mathrm{dl})$ & $0.74 \pm 0.12$ & $0.84 \pm 0.25$ & 0.0995 \\
\hline $\begin{array}{l}\text { eGFR }(\mathrm{mL} / \mathrm{min} / \\
\left.I .73 \mathrm{~m}^{2}\right)\end{array}$ & $80.5 \pm 12.5$ & $74.1 \pm 20.1$ & 0.1764 \\
\hline UA (mg/dl) & $5.7 \pm 1.7$ & $5.9 \pm 1.4$ & 0.5096 \\
\hline $\mathrm{Na}(\mathrm{mEq} / \mathrm{l})$ & $140.7 \pm 3.2$ & $14 \mid .4 \pm 2.2$ & 0.2507 \\
\hline $\mathrm{K}(\mathrm{mEq} / \mathrm{l})$ & $4.3 \pm 0.4$ & $4.3 \pm 0.4$ & $0.664 I$ \\
\hline $\mathrm{Cl}(\mathrm{mEq} / \mathrm{l})$ & $103.5 \pm 3.7$ & $104.8 \pm 2.5$ & 0.9776 \\
\hline WBC $(/ \mu \mathrm{L})$ & $\begin{array}{l}6336.3 \pm \\
1306.0\end{array}$ & $6388.9 \pm 2013.2$ & 0.9112 \\
\hline Neut $(/ \mu \mathrm{L})$ & $4 \mid 70.0 \pm 982.2$ & $3804.3 \pm 1499.4$ & 0.3543 \\
\hline Lymph $(/ \mu \mathrm{L})$ & $1595.3 \pm 770.5$ & $1833.8 \pm 677.6$ & 0.1947 \\
\hline Mono $(/ \mu \mathrm{L})$ & $390.7 \pm 122.5$ & $393.0 \pm 143.8$ & 0.9514 \\
\hline Eos $(/ \mu \mathrm{L})$ & $\begin{array}{l}80.0 \\
(60.0-250.0)\end{array}$ & $\begin{array}{l}170.0 \\
(100.0-260.0)\end{array}$ & 0.0554 \\
\hline Baso $(/ \mu \mathrm{L})$ & $28.0 \pm 15.7$ & $42.8 \pm 23.5$ & 0.0016 \\
\hline $\mathrm{RBC}\left(10^{6} / \mu \mathrm{L}\right)$ & $4.52 \pm 0.42$ & $4.58 \pm 0.54$ & 0.6126 \\
\hline $\mathrm{Hb}(\mathrm{g} / \mathrm{dl})$ & $14.0 \pm 1.2$ & $14.0 \pm 1.6$ & 0.8423 \\
\hline $\mathrm{Ht}(\%)$ & $42.5 \pm 3.6$ & $42.9 \pm 4.3$ & 0.7000 \\
\hline Plt $(104 / \mu \mathrm{L})$ & $20.9 \pm 5.6$ & $22.4 \pm 6.2$ & 0.3176 \\
\hline $\operatorname{lgE}(\mathrm{IU} / \mathrm{mL})$ & $\begin{array}{l}89.3 \\
(30.9-221.0)\end{array}$ & $69.6(23.4-242.0)$ & 0.7387 \\
\hline $\mathrm{Fe}(\mu \mathrm{g} / \mathrm{dl})$ & $96.2 \pm 31.8$ & $93.7 \pm 39.3$ & 0.7947 \\
\hline
\end{tabular}

(Continued)
Table 2 (Continued).

\begin{tabular}{|l|l|l|l|}
\hline & $\begin{array}{l}\text { Developed } \\
\text { Lung Cancer } \\
(\mathbf{n}=19)\end{array}$ & $\begin{array}{l}\text { Did Not Develop } \\
\text { Lung Cancer } \\
(\mathbf{n}=\mathbf{2 0 5})\end{array}$ & P value \\
\hline Fer $(\mathrm{ng} / \mathrm{mL})$ & $\begin{array}{l}\text { III.7 } \\
(61.0-149.5)\end{array}$ & $90.6(43.5-173.5)$ & 0.6470 \\
\hline hsCRP $(\mathrm{mg} / \mathrm{dl})$ & $\begin{array}{l}0.10 \\
(0.04-0.34)\end{array}$ & $0.09(0.04-0.2 \mathrm{I})$ & 0.8805 \\
\hline D-dimer $(\mu \mathrm{g} / \mathrm{dl})$ & $\begin{array}{l}0.86 \\
(0.53-1.59)\end{array}$ & $0.79(0.54-1.36)$ & 0.5744 \\
\hline Fib $(\mathrm{mg} / \mathrm{dl})$ & $329.1 \pm 49.6$ & $329.0 \pm 79.3$ & 0.9946 \\
\hline $5-\mathrm{HIAA}(\mathrm{ng} / \mathrm{mL})$ & $5.3(4.0-9.4)$ & $6.0(4.7-8.5)$ & $0.368 \mathrm{I}$ \\
\hline ACE $(\mathrm{U} / \mathrm{l})$ & $11.2(9.0-15.0)$ & $12.8(10.4-15.8)$ & 0.2570 \\
\hline TARC $(\mathrm{pg} / \mathrm{mL})$ & $397.9 \pm 197.3$ & $384.0 \pm 228.8$ & 0.8310 \\
\hline Hcy $(\mathrm{nmol} / \mathrm{mL})$ & $10.8 \pm 2.7$ & $11.5 \pm 5.3$ & 0.5686 \\
\hline PRA $(\mathrm{ng} / \mathrm{mL} / \mathrm{hr})$ & $1.3(0.6-1.4)$ & $1.2(0.7-2.3)$ & $0.365 \mathrm{I}$ \\
\hline
\end{tabular}

Note: Data are presented as mean $\pm S D$, median (with ranges in parentheses, 25th-75th percentile) or $\mathrm{n}$.

Abbreviations: BMI, body mass index; CAT, COPD assessment score; LAA, low attenuation area on CT scan; FeNO, fractional exhaled nitric oxide; LAMA, longacting muscarinic antagonist; LABA, long-acting $\beta 2$ agonist; ICS, inhaled corticosteroid; ACO, asthma-COPD overlap; IgE, immunoglobulin E; Fer, ferritin; hsCRP, highsensitivity C-reactive protein; Fib, fibrinogen; 5-HIAA, 5-hydroxyindoleacetic acid; TARC, thymus and activation-regulated chemokine; Hcy, homocysteine; PRA, plasma renin activity.

because that was their wish or because they experienced impaired pulmonary function, and of those, three died during the follow-up period. Therefore, in this study, two patients died of lung cancer during follow-up, and the percentage of deaths attributable to lung cancer was $11.1 \%(2 / 18)$.

Finally, we performed univariate and multivariate logistic regression analysis for discriminating the subjects who developed lung cancer (Table 4). Severe emphysema assessed by $\mathrm{CT}$ and current smokers were the significant predicting factor for the patients who developed lung cancer.

\section{Discussion}

In this study, we found 19 newly diagnosed cases of lung cancer in COPD patients during a median observation period of 4.58 years. The lung cancer incidence was $1.85 \% /$ year but has been reported to be $0.8 \%$ to $1.7 \% /$ year in COPD patients; ${ }^{7,8}$ however, recent observational studies in Japan have reported that the incidence ranges from $2.06 \%$ to $2.30 \% /$ year, ${ }^{9,10}$ which indicates that lung cancer incidence is continuing to increase. Our results are consistent with these 
reports. The incidence of lung cancer in the general population has been reported as $0.06 \%-0.12 \% /$ year, ${ }^{6,26}$ while the incidence of lung cancer in COPD patients is thought to be remarkably higher than that in the general population. Several studies have shown that as the degree of emphysema becomes more severe, the incidence of lung cancer increases. ${ }^{9,17,18}$ Our results are consistent with these reports.

In recent years, substantial attention has been focused on whether asthma complications and the use of ICS in COPD patients reduce lung cancer risk. However, data on lung cancer risk in patients with asthma are controversial. An atopic constitution, including asthma, has been suggested to be associated with an increased risk of lung cancer. ${ }^{27,28}$ However, some studies have also reported a reduced risk ${ }^{29}$ or that asthma is not relevant to lung cancer development. ${ }^{30}$ It has also been reported that ICS reduce lung cancer risk in patients with COPD. ${ }^{21,22}$ Some evidence suggests that inflammation may be an important contributing factor in the causal pathway of lung cancer, while the mechanism by which COPD is associated with an increased risk of lung cancer is not well established. ${ }^{22}$ Parimon et al hypothesized that ICS reduces airway inflammation, which results in decreased cell turnover; this in turn lowers the risk for propagation of genetic errors. ${ }^{31}$ We also found that the individuals who did not develop lung cancer were more greater ICS use during the follow-up period. In addition, individuals who did not develop lung cancer were more likely to have ACO, although there was no significant difference. Therefore, ICS may have been effective in suppressing lung cancer development (Table 2).

It has been previously reported that squamous cell carcinomas comprise a majority of COPD-associated lung cancers. ${ }^{8,32}$ However, observational studies conducted in Japan have reported that adenocarcinoma is the most common histologic lung cancer type associated with COPD,${ }^{9}$ which is in agreement with the present study. Where it is possible that the difference arises from a mutation in a gene such as epidermal growth factor receptor (EGFR), it is unclear whether this difference is due to race or some other patient characteristic. However, data on genetic mutations were not collected for this study, and thus their involvement is unclear and cannot be speculated. Only one patient was treated with cytotoxic chemotherapy because the results of gene mutation were negative. For other patients, appropriate treatments such as surgery, radiation therapy, and symptomatic treatment were selected according to the staging of the lung cancer and their general condition. Roviello et al reviewed studies of the therapeutic effects of molecular-targeted drugs in elderly patients with advanced lung cancer. ${ }^{33}$ In this metaanalysis, EGFR tyrosine kinase inhibitors (TKIs) showed a significant effect in suppressing disease progression in elderly patients with advanced lung cancer. According to these results, we should consider EGFR TKIs as the effective treatment options in elderly patients with advanced

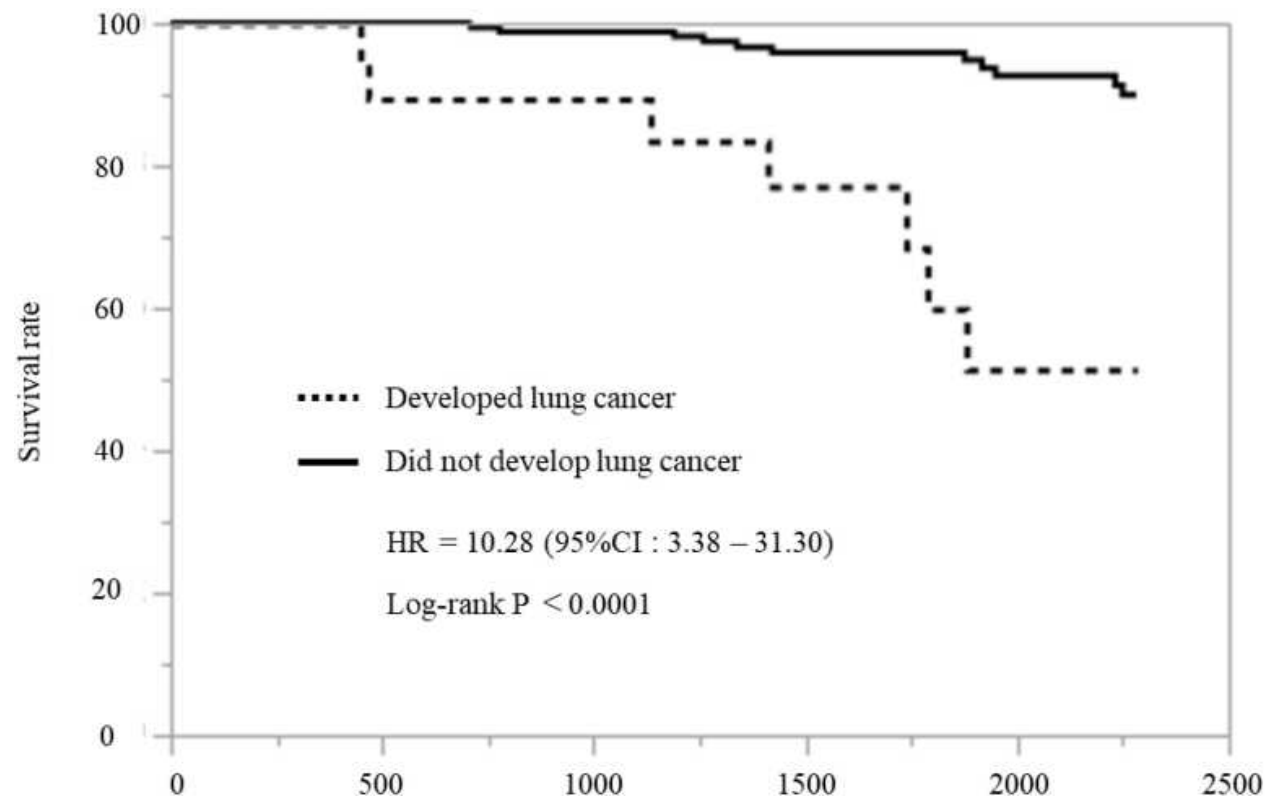

Figure 2 Survival curves. A survival curve analysis according to the Kaplan-Meier method showed that mortality was significantly increased in the lung cancer development group compared with the group that did not develop lung cancer. 
Table 3 Characteristics of Patients Who Developed Lung Cancer

\begin{tabular}{|c|c|c|c|c|c|c|}
\hline & Histology & $\begin{array}{l}\text { Days Before } \\
\text { Onset }\end{array}$ & Stage & Therapy & Outcome & Supplement \\
\hline I. 6I-yr M & Ad & 41 & $\begin{array}{l}\text { PTIaNOMO } \\
\text { IAI }\end{array}$ & Ope & Survival & \\
\hline 2. $66-\mathrm{yr} \mathrm{M}$ & Ad & 1042 & $\begin{array}{l}\text { PTIbNOMO } \\
\text { IA2 }\end{array}$ & Ope & Survival & \\
\hline 3. 78-yr M & Ad & 931 & $\begin{array}{l}\text { cTIaNOMO } \\
\text { IAI }\end{array}$ & RT & Survival & \\
\hline 4. $65-y r M$ & Ad & 247 & $\begin{array}{l}\text { cT3N2M0 } \\
\text { IIIB }\end{array}$ & Chemo & Survival & \\
\hline 5. 66-yr M & Ad & 1133 & $\begin{array}{l}\text { cTIbNOMO } \\
\text { IA2 }\end{array}$ & RT & Survival & \\
\hline 6. $74-y r ~ M$ & Ad & 1918 & $\begin{array}{l}\text { cTIbNOMO } \\
\text { IA2 }\end{array}$ & Ope & Survival & Complication of interstitial pneumonia \\
\hline 7. 76-yr M & $\mathrm{Sq}$ & 181 & $\begin{array}{l}\text { PTIaNOMO } \\
\text { IAI }\end{array}$ & Ope & Survival & \\
\hline 8. $67-y r M$ & $\mathrm{Sq}$ & 392 & $\begin{array}{l}\text { PTIbNOMO } \\
\text { IA2 }\end{array}$ & Ope & Survival & Complication of interstitial pneumonia \\
\hline 9. 67-yr M & $\mathrm{Sq}$ & 929 & $\begin{array}{l}\text { PTIbNOMO } \\
\text { IA2 }\end{array}$ & Ope & Survival & \\
\hline 10. $78-y r ~ M$ & Non-small & 95 & $\begin{array}{l}\text { PTIbNOMO } \\
\text { IA2 }\end{array}$ & Ope & Survival & \\
\hline II. $70-y r ~ M$ & Small & 317 & $\begin{array}{l}\text { cT3N2M0 } \\
\text { IIIB }\end{array}$ & Chemo & $\begin{array}{l}\text { Death (Lung } \\
\text { cancer) }\end{array}$ & \\
\hline 12. $68-y r ~ M$ & Small & 1019 & $\begin{array}{l}\text { cT3N2M0 } \\
\text { шB }\end{array}$ & BSC & $\begin{array}{l}\text { Death (Interstitial } \\
\text { pneumonia) }\end{array}$ & Complication of interstitial pneumonia \\
\hline 13. $80-y r ~ M$ & Clinical & 941 & $\begin{array}{l}\text { cTIaNOMO } \\
\text { IAI }\end{array}$ & RT & Death (Unknown) & \\
\hline 14. 77-yr M & Clinical & 968 & $\begin{array}{l}\text { cTIaNOMO } \\
\text { IAI }\end{array}$ & RT & Death (Unknown) & \\
\hline I5. $78-y r ~ M$ & Clinical & 217 & $\begin{array}{l}\text { cT2aNOMIa } \\
\text { IVA }\end{array}$ & BSC & Death (Senility) & $\begin{array}{l}\text { Due to poor pulmonary function, treatment } \\
\text { could not be performed }\end{array}$ \\
\hline 16. $79-y r ~ M$ & Clinical & 643 & $\begin{array}{l}\text { CT2aNOMO } \\
\text { IB }\end{array}$ & BSC & $\begin{array}{l}\text { Death (Lung } \\
\text { cancer) }\end{array}$ & $\begin{array}{l}\text { The patient did not want to be examined and } \\
\text { treated }\end{array}$ \\
\hline 17. 86-yr M & Clinical & 817 & $\begin{array}{l}\text { cTIaNOMO } \\
\text { IAI }\end{array}$ & BSC & Survival & $\begin{array}{l}\text { The patient did not want to be examined and } \\
\text { treated }\end{array}$ \\
\hline 18. 6I-yr M & Clinical & 1444 & $\begin{array}{l}\text { cT3NOMO } \\
\text { IIIB }\end{array}$ & BSC & Survival & $\begin{array}{l}\text { Due to poor pulmonary function, treatment } \\
\text { could not be administered }\end{array}$ \\
\hline 19. 77-yr M & Clinical & 1500 & $\begin{array}{l}\text { cTIaNOMO } \\
\text { IAI }\end{array}$ & BSC & $\begin{array}{l}\text { Death (Pancreatic } \\
\text { cancer) }\end{array}$ & $\begin{array}{l}\text { The patient did not want to be examined and } \\
\text { treated }\end{array}$ \\
\hline
\end{tabular}

Abbreviations: Ad, adenocarcinoma; Sq, squamous cell carcinoma; Small, small cell carcinoma; Ope, operation; RT, radiation therapy; Chemo, chemotherapy; BSC, best supportive care. 
Table 4 Univariate and Multivariate Logistic Regression Analyses for Discriminating the Subjects Who Developed Lung Cancer

\begin{tabular}{|l|l|l|l|}
\hline Univariate Analysis & OR & $95 \%$ CI & $\mathbf{P}$ \\
\hline Age (increasing I-age) & 1.03 & $0.98-1.10$ & 0.2717 \\
\hline LAA (increasing I-score) & 1.14 & $1.00-1.29$ & 0.0493 \\
\hline Current smoker (vs non-smoker) & 3.65 & $1.27-10.54$ & 0.0253 \\
\hline GOLD II (vs I) & 1.53 & $0.51-4.56$ & 0.4453 \\
\hline GOLD III (vs I) & 3.39 & $0.40-28.77$ & 0.2625 \\
\hline GOLD IV (vs I) & 0.16 & $0.04-0.70$ & 0.0153 \\
\hline Multivariate Analysis & $\mathbf{O R}$ & $95 \%$ CI & $\mathbf{P}$ \\
\hline Age (increasing I-age) & 1.07 & $0.97-I .18$ & 0.1097 \\
\hline LAA (increasing I-score) & 1.17 & $1.01-1.37$ & 0.0399 \\
\hline Current smoker (vs non-smoker) & 4.8 & $1.15-20.46$ & 0.0416 \\
\hline GOLD II (vs I) & 2.74 & $0.71-10.63$ & 0.1435 \\
\hline GOLD III (vs I) & - & - & 0.9986 \\
\hline GOLD IV (vs I) & 0.38 & $0.05-2.66$ & 0.3273 \\
\hline
\end{tabular}

Abbreviation: LAA, low attenuation area on CT scan.

lung cancer. Fathi et al reviewed studies for the investigation of gene mutations in patients with lung cancer. ${ }^{34}$ As a result, it was shown that typical carcinogenic genes such as EGFR and echinoderm microtubule-associated proteinlike 4 anaplastic lymphoma kinase (EML4-ALK), and various DNA repair genes were mainly investigated. If we can analyze carcinogenic genes of lung cancer, it is expected to be very useful information to know the carcinogenesis in patients with COPD. However, we usually do not analyze oncogenes in patients with COPD before they develop lung cancer. Even if we diagnose a patient with lung cancer, analysis of all carcinogenic genes is expensive and uncommon. When the patients with COPD can be screened before carcinogenesis and the carcinogenic genes can be identified, it will be possible to understand the future risk of patients with COPD. We think it is necessary to proceed with future research on these findings.

Lung cancer is known to have a negative impact on the prognosis of COPD patients, ${ }^{11}$ and similarly, in this study, the mortality rate was significantly higher in the group that developed lung cancer (Table 2, Figure 2). The proportion of deaths attributed to lung cancer was $11.1 \%$, which was similar to the previously reported range of $12-14 \% .^{12-14}$ Lung cancer complications are believed to be one of the important complications because they lead to a worse prognosis in patients with COPD. In this study, patients with non-small cell lung cancer received the appropriate therapy, and none of those patients died during the followup period. We need to recognize that patients with COPD have an increased risk of developing lung cancer. We should make efforts to detect lung cancer earlier by conducting regular examinations such as CT. Although it is difficult to predict the development of lung cancer in patients with COPD, annual CT screening may detect lung cancer at an early stage and lead to appropriate treatment.

Including the many uncertainties about the factors that predict lung cancer development in COPD patients, this study has several limitations. Similarly, this study could not identify factors that predict lung cancer development, with the exception of smoking and degree of emphysema (Table 4). Therefore, in COPD patients, it is necessary to always pay attention to lung cancer development and to consider it a possibility. This study was a prospective study and was conducted at a single hospital, and thus, the sample size is small. Additionally, the median observation period in this study was 4.58 years, which was less than 5 years. Longer observations may be needed to observe the event of carcinogenesis. Therefore, our findings should be validated by a new prospective study with an expanded sample size.

\section{Conclusion}

In patients with COPD, the incidence of lung cancer is higher, and the development of lung cancer leads to a worse prognosis; however, since lung cancer development is unpredictable, attention should be paid to all patients. Annual CT screening might be useful in diagnosing lung cancer earlier staging and providing appropriate treatment.

\section{Acknowledgments}

The authors would like to thank Enago for the English language review.

\section{Author Contributions}

All authors contributed to data analysis, drafting or revising the article, have agreed on the journal to which the article will be submitted, gave final approval of the version to be published, and agree to be accountable for all aspects of the work. 


\section{Funding}

This research was funded by CHUGAI PHARMA CEUTICAL CO., LTD.

\section{Disclosure}

HMa received lecture fees from Boehringer Ingelheim Japan. SI received lecture fees from Otsuka Pharmaceutical Co., Ltd, Novartis Pharma K.K., AstraZeneca K.K., GlaxoSmithKline K.K., Chugai Pharmaceutical Co., Ltd., Boehringer Ingelheim Japan, MSD K.K., ONO PHARMACEUTICAL CO., LTD., Meiji Seika Pharma Co., Ltd., KYORIN Pharmaceutical Co., Ltd., Taiho Pharmaceutical Co., Ltd., Eli Lilly Japan K.K., NIHON PHARMACEUTICAL CO., LTD., TEIJIN PHARMA LIMITED, Pfizer Japan Inc., DAIICHI SANKYO COMPANY, LIMITED, and received research grant from Chugai Pharmaceutical Co., Ltd., Novartis Pharma K.K., MSD K.K., KYORIN Pharmaceutical Co., Ltd., and JSPS KAKENHI (grant number 19K08658). YS received lecture fees from Boehringer Ingelheim Japan, AstraZeneca K.K., Novartis Pharma K.K., KYORIN Pharmaceutical Co., Ltd., Chugai Pharmaceutical Co., Ltd., Pfizer Japan Inc., GlaxoSmithKline K.K., CHEST, received advisory fee from GlaxoSmithKline K.K., and received research grant from Boehringer Ingelheim Japan, AstraZeneca K.K., Novartis Pharma K.K., KYORIN Pharmaceutical Co., Ltd., Chugai Pharmaceutical Co., Ltd., and JSPS KAKENHI (grant number 19K08658). TK received lecture fee from ONO PHARMACEUTICAL CO., LTD. and research grant from JSPS KAKENHI (Grant Number JP 15K19411). TO received lecture fee from MSD K.K. KF received lecture fee from Boehringer Ingelheim Japan. SY received lecture fees from Boehringer Ingelheim Japan, AstraZeneca K.K. $\mathrm{HN}$ received lecture fees from Pfizer Japan Inc., Boehringer Ingelheim Japan, AstraZeneca K.K. KS received lecture fee from Chugai Pharmaceutical Co., Ltd., GlaxoSmithKline K.K., AstraZeneca K.K., Sanofi K.K. and research grant from GlaxoSmithKline K.K. MS received lecture fees from AstraZeneca K.K., Boehringer Ingelheim Japan. TN received lecture fee from Teijin Pharma Ltd Japan. KY received lecture fee from Pfizer Japan Inc. AI received lecture fees from AstraZeneca K. K., Boehringer Ingelheim Japan. MW received research grants from MSD K.K., Asahi Kasei Pharma Corporation., Asahi Kasei Medical Co., Ltd., KISSEI PHARMACEUTICAL CO., LTD., Kyowa Kirin Co.,
Ltd., Sanofi K.K., SHIONOGI \& CO., LTD., Taisho Pharma Co., Ltd., CHUGAI PHARMACEUTICAL CO., LTD., TEIJIN PHARMA LIMITED, Torii Pharmaceutical Co., Ltd., Nihon Medi-Physics Co., Ltd., Novartis Pharma K.K., BIOTRONIK Japan, Inc., FUJIFILM Toyama Chemical Co., Ltd., Roche Diagnostics K.K., Kowa Company, Limited, Abbott Vascular Japan Co., Ltd., Cardinal Health Japan, Kowa Company, Limited, research grants and personal fees from Actelion Pharmaceuticals Japan Ltd., Astellas Pharma Inc., Otsuka Pharmaceutical Co., Ltd., ONO PHARMACEUTICAL CO., LTD., DAIICHI SANKYO COMPANY, LIMITED, Sumitomo Dainippon Pharma Co., Ltd., Takeda Pharmaceutical Company Limited, Nippon Boehringer Ingelheim Co., Ltd., MEDTRONIC JAPAN CO., LTD., Bayer Yakuhin, Ltd., Pfizer Japan Inc., MOCHIDA PHARMACEUTICAL CO., LTD., personal fees from Mitsubishi Tanabe Pharma Corporation, KOWA PHARMACEUTICAL COMPANY LTD., TOA EIYO LTD., Bristol-Myers Squibb K.K., AstraZeneca K.K., Edwards Lifesciences Corporation., Amgen. Inc., Japan Lifeline Co., Ltd. The authors report no other conflicts of interest in this work.

\section{References}

1. World Health Organization. The top 10 causes of death; 24 May 2018. Available from: http://who.int/mediacentre/factsheets/fs310/en/. Accessed May 3, 2020.

2. MacNee W. Pathology, pathogenesis, and pathophysiology. BMJ. 2006;332(7551):1202-1204. doi:10.1136/bmj.332.7551.1202

3. Bradford E, Jacobson S, Varasteh J, et al. The value of blood cytokines and chemokines in assessing COPD. Respir Res. 2017;18(1):180. doi:10.1186/s12931-017-0662-2

4. Sin DD, Anthonisen NR, Soriano JB, Agust AG. Mortality in COPD: role of comorbidities. Eur Respir J. 2006;28(6):1245-1257. doi:10.1183/09031936.00133805

5. Young RP, Hopkins RJ, Christmas T, Black PN, Metcalf P, Gamble GD. COPD prevalence is increased in lung cancer, independent of age, sex and smoking history. Eur Respir J. 2009;34 (2):380-386. doi:10.1183/09031936.00144208

6. Mannino DM, Aguayo SM, Petty TL, Redd SC. Low lung function and incident lung cancer in the United States: data from the First National Health and Nutrition examination survey follow-up. Arch Intern Med. 2003;163(12):1475-1480. doi:10.1001/ archinte.163.12.1475

7. Skillrud DM, Offord KP, Miller RD. Higher risk of lung cancer in chronic obstructive pulmonary disease. A prospective, matched, controlled study. Ann Intern Med. 1986;105(4):503-507. doi:10.7326/ 0003-4819-105-4-503

8. de Torres JP, Marín JM, Casanova C, et al. Lung cancer in patients with chronic obstructive pulmonary disease- incidence and predicting factors. Am J Respir Crit Care Med. 2011;184(8):913-919. doi:10.1164/rccm.201103-0430OC

9. Chubachi S, Takahashi S, Tsutsumi A, et al. Radiologic features of precancerous areas of the lungs in chronic obstructive pulmonary disease. Int J Chron Obstruct Pulmon Dis. 2017;12:1613-1624. 
10. Haruna A, Muro S, Nakano Y, et al. CT scan findings of emphysema predict mortality in COPD. Chest. 2010;138(3):635-640. doi:10.1378/chest.09-2836

11. Smith MC, Wrobel JP. Epidemiology and clinical impact of major comorbidities in patients with COPD. Int J Chron Obstruct Pulmon Dis. 2014;9:871-888. doi:10.2147/COPD.S49621

12. Vilkman S, Keistinen T, Tuuponen T, Kivelä SL. Survival and cause of death among elderly chronic obstructive pulmonary disease patients after first admission to hospital. Respiration. 1997;64 (4):281-284. doi:10.1159/000196687

13. Bale G, Martínez-Camblor P, Burge PS, Soriano JB. Long-term mortality follow-up of the ISOLDE participants: causes of death during 13 years after trial completion. Respir Med. 2008;102 (10):1468-1472. doi:10.1016/j.rmed.2008.04.001

14. Jenkins CR, Jones PW, Calverley PM, et al. Efficacy of salmeterol/ fluticasone propionate by GOLD stage of chronic obstructive pulmonary disease: analysis from the randomised, placebo-controlled TORCH study. Respir Res. 2009;10(1):59. doi:10.1186/1465-9921-10-59

15. Anthonisen NR, Skeans MA, Wise RA, Manfreda J, Kanner RE, Connett JE; Lung Health Study Research Group. The effects of a smoking cessation intervention on 14.5-year mortality: a randomized clinical trial. Ann Intern Med. 2005;142(4):233-239. doi:10.7326/0003-4819-142-4-200502150-00005

16. Shibata Y, Inoue S, Igarashi A, et al. A lower level of forced expiratory volume in 1 second is a risk factor for all-cause and cardiovascular mortality in a Japanese population: the Takahata study. PLoS One. 2003;8(12):e83725. doi:10.1371/journal.pone.0083725

17. de Torres JP, Bastarrika G, Wisnivesky JP, et al. Assessing the relationship between lung cancer risk and emphysema detected on low-dose CT of the chest. Chest. 2007;132(6):1932-1938. doi: $10.1378 /$ chest.07-1490

18. Wilson DO, Weissfeld JL, Balkan A, et al. Association of radiographic emphysema and airflow obstruction with lung cancer. Am J Respir Crit Care Med. 2008;178(7):738-744. doi:10.1164/ rccm.200803-435OC

19. Wasswa-Kintu S, Gan WO, Man SFP, Pare PD, Sin DD. Relationship between reduced forced expiratory volume in one second and the risk of lung cancer: a systematic review and meta-analysis. Thorax. 2005;60(7):570-575. doi:10.1136/thx.2004.037135

20. Zulueta JJ, Wisnivesky JP, Henschke CI, et al. Emphysema scores predict death from COPD and lung cancer. Chest. 2012;141 (5):1216-1223. doi:10.1378/chest.11-0101

21. Sandelin M, Mindus S, Thuresson M, et al. Factors associated with lung cancer in COPD patients. Int $J$ Chron Obstruct Pulmon Dis. 2018;13:1833-1839.

22. Raymakers AJN, Sadatsafavi M, Sin DD, FitzGerald JM, Marra CA, Lynd LD. Inhaled corticosteroids and the risk of lung cancer in COPD: a population-based cohort study. Eur Respir J. 2019;53 (6):1801257. doi:10.1183/13993003.01257-2018
23. The Japanese Respiratory Society. [Guidelines for the management of asthma and COPD overlap]. Tokyo: Medical Review; 2018. Japanese.

24. Ishii A, Sugiyama H, Kirikae T, et al. Correlations between computed tomography images and respiratory function in chronic obstructive pulmonary disease. AJRS. 2014;3(2):221-226.

25. The Japan Lung Cancer Society. [General rule for clinical and pathological record of lung cancer]. Tokyo: KANEHARA \& CO; 2017. Japanese.

26. Smith BD, Smith GL, Hurria A, Hortobagyi GN, Buchholz TA. Future of cancer incidence in the United States: burdens upon an aging, changing nation. J Clin Oncol. 2009;27(17):2758-2765. doi:10.1200/JCO.2008.20.8983

27. Santillan AA, Camargo CA Jr, Colditz GA. A meta-analysis of asthma and risk of lung cancer (United States). Cancer Causes Control. 2003;14(4):327-334. doi:10.1023/A:1023982402137

28. Gorlova OY, Zhang Y, Schabath MB, Lei L, Zhang Q, Amos CI. Never smokers and lung cancer risk: a case-control study of epidemiological factors. Int $J$ Cancer. 2006;118(7):1798-1804. doi:10.1002/ijc. 21561

29. El-Zein M, Parent ME, Kâ K, Siemiatycki J, St-Pierre Y, Rousseau MC. History of asthma or eczema and cancer risk among men: a population-based case-control study in Montreal, Quebec, Canada. Ann Allergy Asthma Immunol. 2010;104(5):378-384. doi:10.1016/j.anai.2010.03.003

30. Ramanakumar AV, Parent ME, Menzies D, Siemiatycki J. Risk of lung cancer following nonmalignant respiratory conditions: evidence from two case-control studies in Montreal, Canada. Lung Cancer. 2006;53(1):5-12. doi:10.1016/j.lungcan.2006.04.007

31. Parimon T, Chien JW, Bryson CL, McDonell MB, Udris EM, Au DH. Inhaled corticosteroids and risk of lung cancer among patients with chronic obstructive pulmonary disease. Am J Respir Crit Care Med. 2007;175(7):712-719. doi:10.1164/rccm.200608-1125OC

32. Papi A, Casoni G, Caramori G, et al. COPD increases the risk of squamous histological subtype in smokers who develop non-small cell lung carcinoma. Thorax. 2004;59(8):679-681. doi:10.1136/ thx.2003.018291

33. Roviello G, Zanotti L, Cappelletti MR, et al. Are EGFR tyrosine kinase inhibitors effective in elderly patients with EGFR-mutated non-small cell lung cancer? Clin Exp Med. 2018;18(1):15-20. doi:10.1007/s10238-017-0460-7

34. Fathi Z, Syn NL, Zhou JG, Roudi R. Molecular epidemiology of lung cancer in Iran: implications for drug development and cancer prevention. J Hum Genet. 2018;63(7):783-794. doi:10.1038/s10038018-0450-y

\section{Publish your work in this journal}

The International Journal of COPD is an international, peer-reviewed journal of therapeutics and pharmacology focusing on concise rapid reporting of clinical studies and reviews in COPD. Special focus is given to the pathophysiological processes underlying the disease, intervention programs, patient focused education, and self management protocols. This journal is indexed on PubMed Central, MedLine and CAS. The manuscript management system is completely online and includes a very quick and fair peer-review system, which is all easy to use. Visit http://www.dovepress.com/testimonials.php to read real quotes from published authors. 